\title{
LETTER
}

\section{W continuous-wave Ho:YAG single-crystal fiber laser}

\author{
Yongguang Zhao ${ }^{\oplus 1,2}$, Li Wang ${ }^{1}$, Weidong $\mathrm{Chen}^{1}$, Jianlei Wang ${ }^{2}$, Qingsong Song ${ }^{2}$, Xiaodong $\mathrm{Xu}^{2}$, \\ Ying $\mathrm{Liu}^{2}$, Deyuan $\mathrm{Shen}^{2}$, Jun $\mathrm{Xu}^{3}$, Xavier Mateos ${ }^{4}$, Pavel Loiko ${ }^{5}$, Zhengping Wang ${ }^{6}$, Xinguang $\mathrm{Xu}^{6}$, \\ Uwe Griebner $^{1}$, and Valentin Petrov ${ }^{1}$ \\ ${ }^{1}$ Max Born Institute for Nonlinear Optics and Short Pulse Spectroscopy, Max-Born-Str. 2a, D-12489 Berlin, Germany \\ ${ }^{2}$ Jiangsu Key Laboratory of Advanced Laser Materials and Devices, Jiangsu Normal University, Xuzhou 221116, China \\ ${ }^{3}$ School of Physics Science and Engineering, Institute for Advanced Study, Tongji University, Shanghai 200092, China \\ ${ }^{4}$ Departament Química Física i Inorgànica, Física i Cristal.lografia de Materials i Nanomaterials (FiCMA-FiCNA)-EMaS, Universitat \\ Rovira i Virgili, Campus Sescelades, E-43007 Tarragona, Spain \\ ${ }^{5}$ Centre de Recherche sur les Ions, les Matériaux et la Photonique (CIMAP), UMR 6252 CEA-CNRS-ENSICAEN, Université de Caen, \\ 6 Boulevard du Maréchal Juin, 14050 Caen Cedex 4, France \\ ${ }^{6}$ State Key Laboratory of Crystal Materials and Institute of Crystal Materials, Shandong University, Jinan 250100, China \\ (Received 7 April 2020; revised 15 May 2020; accepted 20 May 2020)
}

\begin{abstract}
We report on a high-power Ho:YAG single-crystal fiber (SCF) laser inband pumped by a high-brightness Tm-fiber laser at $1908 \mathrm{~nm}$. The Ho:YAG SCF grown by the micro-pulling-down technique exhibits a propagation loss of $0.05 \pm 0.005 \mathrm{~cm}^{-1}$ at $2.09 \mu \mathrm{m}$. A continuous-wave output power of $35.2 \mathrm{~W}$ is achieved with a slope efficiency of $42.7 \%$, which is to the best of our knowledge the highest power ever reported from an SCF-based laser in the $2 \mu \mathrm{m}$ spectral range.
\end{abstract}

Keywords: high-power laser; Ho:YAG; mid-IR laser; single-crystal fiber

\section{Introduction}

High-power solid-state lasers in the $2 \mu \mathrm{m}$ spectral region are actively investigated due to their widespread applications in various fields such as eye-safe coherent laser radar for longrange hard-target measurement ${ }^{[1]}$, hard or soft tissue ablation owing to strong laser absorption by water molecules ${ }^{[2,3]}$, high-sensitivity global wind and active atmospheric remote sensing ${ }^{[4]}$, and pumping optical parametric oscillators for the development of mid-IR sources ${ }^{[5]}$. Current concepts for the power scaling of such $2 \mu \mathrm{m}$ laser sources tend to use Tmor Ho-doped glass fibers ${ }^{[6,7]}$. However, stimulated Brillouin scattering, stimulated Raman scattering, optical damage and photo-darkening present serious limiting factors ${ }^{[8]}$. Singlecrystal fibers (SCFs), typically with a diameter below $1 \mathrm{~mm}$ and a length of a few centimeters, are an alternative for highpower laser systems owing to the fiber-like geometry, higher

Correspondence to: Y. Zhao, Max Born Institute for Nonlinear Optics and Short Pulse Spectroscopy, Max-Born-Str. 2a, D-12489 Berlin, Germany. Email: yongguangzhao@yeah.net thermal conductivity, higher optical damage threshold and much lower Brillouin gain coefficient ${ }^{[9]}$. The amplification of 800-fs pulses to an average power of $160 \mathrm{~W}^{[10]}$ and $380-$ fs pulses to a peak power of $2.2 \mathrm{GW}^{[11]}$ using $\mathrm{Yb}$ :YAG SCFs indicates the inherent capability for simultaneous handling of high average and peak powers. With good thermal management provided by the large surface-to-volume ratio (i.e., small temperature difference between the center and the edge), output powers in the continuous-wave (CW) regime of $34 \mathrm{~W}$ at $0.95 \mu \mathrm{m}$ and $>250 \mathrm{~W}$ at $1 \mu \mathrm{m}$ have been, respectively, demonstrated with Nd-doped ${ }^{[12]}$ and $\mathrm{Yb}$-doped YAG SCFs ${ }^{[13]}$, in which the high overlap between the pump and the laser beam was brought about by pump guiding. Alternatively, high beam quality pump sources (e.g., highbrightness laser diodes or fiber lasers offering a sufficiently high depth of focus to cover the entire SCF) make this possible for freely propagating pumps. In comparison to a waveguide pumped Yb:YAG SCF, a conventional Yb:YAG rod with an unguided pump using a high-brightness laser diode has been demonstrated to exhibit even a higher gain in

(C) The Author(s), 2020. Published by Cambridge University Press in association with Chinese Laser Press. This is an Open Access article, distributed under the terms of the Creative Commons Attribution licence (http://creativecommons.org/ licenses/by/4.0/), which permits unrestricted re-use, distribution, and reproduction in any medium, provided the original work is properly cited. 
a two-pass chirped pulse amplifier ${ }^{[14]}$. On the other side, Tmor Ho-SCF based laser systems in the $2 \mu \mathrm{m}$ spectral range have been seldom reported. In 2016, such a laser at $2.09 \mu \mathrm{m}$ was demonstrated for the first time employing a Ho:YAG SCF fabricated by laser heated pedestal growth (LHPG) ${ }^{[15]}$. A quasi-CW output power of $23.5 \mathrm{~W}$ was obtained under pulsed pumping. However, output power roll-off was observed at $\sim 10 \mathrm{~W}$ in the pure $\mathrm{CW}$ regime. Most recently, we have reported on the first $Q$-switched Ho:YAG SCF laser delivering $7.5 \mathrm{~ns}$ pulses with $0.19 \mathrm{MW}$ peak power ${ }^{[16]}$. No optical damage or spectral broadening was observed in this experiment, indicating the high damage resistivity and suppressed nonlinear effects in the SCF. Nevertheless, the $\mathrm{CW}$ laser power of $2 \mu \mathrm{m}$ SCF oscillators/amplifiers was clamped to $\sim 10 \mathrm{~W}$ due to either available pump power or thermal issues under rigid focus conditions for realizing waveguide pumping.

The laser performance in the $\mathrm{CW}$ regime is investigated in the present work by utilizing a high-brightness Tm-fiber laser at $1908 \mathrm{~nm}$ for the resonant pumping of a Ho:YAG SCF grown by the micro-pulling-down ( $\mu$-PD) method. The laser power of $35 \mathrm{~W}$ produced at $2.09 \mu \mathrm{m}$ is to the best of our knowledge the highest power ever reported from an SCFbased laser in the $2 \mu \mathrm{m}$ spectral range.

\section{Experimental details}

YAG $\left(\mathrm{Y}_{3} \mathrm{Al}_{5} \mathrm{O}_{12}\right)$ is the most stable garnet phase in the $\mathrm{Y}_{2} \mathrm{O}_{3}-\mathrm{Al}_{2} \mathrm{O}_{3}$ binary equilibrium diagram and melts congruently around $\sim 1980^{\circ} \mathrm{C}$. Because of the small difference in the ionic radius between $\mathrm{Ho}^{3+}(104.1 \mathrm{pm})$ and $\mathrm{Y}^{3+}$ $(104 \mathrm{pm})$, it is possible to substitute a high level of $\mathrm{Y}^{3+}$ by $\mathrm{Ho}^{3+}$ and the segregation issue is a minor problem in comparison to other dopants such as $\mathrm{Nd}^{3+}(112.3 \mathrm{pm})$ or $\mathrm{Ce}^{3+}(115 \mathrm{pm})$, thus maintaining the advantageous properties of the host crystal. The YAG SCF doped with $0.6 \%$ (atomic fraction) $\mathrm{Ho}^{3+}$ was grown by the $\mu$-PD technique at a temperature slightly higher than the melting point. The fabricated SCF with a total length of $160 \mathrm{~mm}$ was cut into several segments and a 40-mm-long segment was used as a laser element in the present work. Its end faces were polished but left uncoated, whereas the lateral surface was not polished since it is inherently smooth. Figure 1(a) displays a photograph of the end facet of the SCF. The transverse cross-section presents a noncircular shape with a diameter of 960 and $920 \mu \mathrm{m}$ in the two orthotropic directions, which was formed from the natural facets along the $<111>$ direction during the growth process. This growth direction corresponds to the lowest surface energy. The growth of YAG fibers along it results in six facets with hexagonal shape, and this direction is considered to be the easiest growth orientation. To mitigate the thermal load, the SCF, tightly mounted on a specially designed copper holder, was water-cooled to $15.0^{\circ} \mathrm{C}$.

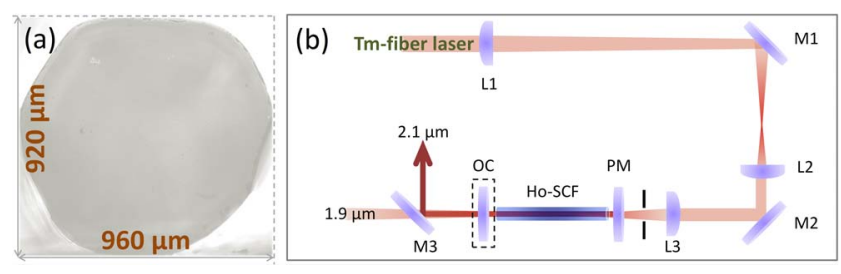

Figure 1. (a) Photograph of the end facet of the Ho:YAG SCF. (b) Schematic of the Ho:YAG SCF laser: L1-L3, lenses with $f=750,100$ and $150 \mathrm{~mm}$, respectively; M1, M2, bending mirrors; M3, dichroic mirror; PM, pump mirror; OC, output coupler.

Figure 1(b) shows the schematic of the Ho:YAG SCF laser. The pump beam emitted from a Tm-fiber laser (IPGTLM-100 W-1908 nm, $\left.M^{2}=1.1\right)$ passed through a $7.5: 1$ telescope system and thereafter was focused by a lens $(f=$ $150 \mathrm{~mm}$ ) into the SCF with a spot diameter of $450 \mu \mathrm{m}$. The beam diameter along the entire SCF was measured to be less than $600 \mu \mathrm{m}$, i.e., smaller than the aperture of the SCF. A plane mirror coated for high reflectivity at the laser wavelength was used as a pump mirror (PM). Plane mirrors with transmission of $T_{\mathrm{oc}}=30 \%, 50 \%, 60 \%, 72 \%$ and 92\% (end-face of the SCF provided 8\% Fresnel reflection at $2.1 \mu \mathrm{m}$ ) were used as output couplers (OCs). A dichroic mirror (M3) was used to separate the laser and pump beams.

\section{Results and discussion}

High-power CW operation of the Ho:YAG SCF laser was studied at a physical cavity length of $45 \mathrm{~mm}$ using different OCs. The low signal single-pass absorption efficiency of the SCF under nonlasing conditions amounted to $94.7 \%$ at $1.9 \mathrm{~W}$ of incident pump power. Further increasing the pump power to $71.3 \mathrm{~W}$, the absorption efficiency dropped to $82.5 \%$ due to the bleaching effect. Under lasing conditions with $T_{\mathrm{oc}}=92 \%$, the corresponding absorption efficiencies for the same pump levels were $95.4 \%$ and $87 \%$. Figure 2(a) shows the output laser performance with respect to the absorbed pump power, and the laser beams in all the cases were measured to be unpolarized. A maximum output power of $35.2 \mathrm{~W}$ was achieved with $T_{\mathrm{oc}}=72 \%$. No power rollover was observed and further power scaling was limited just by the available pump power. The good laser performance is attributed to the high gain and the weak thermal load benefiting from the large surface area-to-volume ratio, i.e., the fiber-like geometry of the SCF. The highest slope efficiency of $53.3 \%$ was achieved by using the end facet of the SCF as OC, i.e., $T_{\mathrm{oc}}=92 \%$. Unfortunately, further power scaling in this case was limited by a careless scratch point at one end facet of the SCF during the experiment. Nevertheless, the straightforward power scaling with almost linear pump power dependence indicates the huge potential of the SCF for high-power laser operation. Laser emission was not achieved without the input mirror, i.e., in a cavity formed by both end facets of the SCF in which case a much 

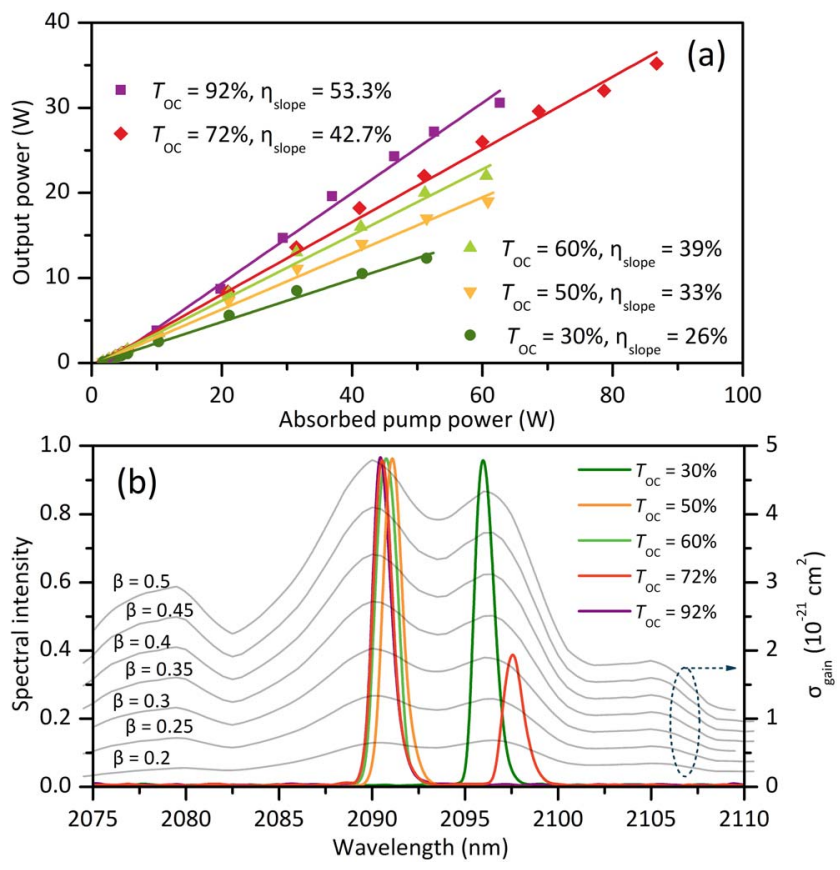

Figure 2. (a) Laser performance of the Ho:YAG SCF laser with different OCs and (b) the corresponding optical spectra. The gray curves are the calculated gain spectra of Ho:YAG ${ }^{[17]}$ with different population inversion parameters $\beta$.

higher population inversion is required. This is an indication of the nonparallel faces of the used SCF. Figure 2(b) shows the optical spectra of the Ho:YAG SCF laser with different OCs (for comparison the gain spectra of $\mathrm{Ho}: \mathrm{YAG}^{[17]}$ are also depicted). The peak wavelength of the laser emission changed from $2090.3 \mathrm{~nm}$ to $2095.7 \mathrm{~nm}$ with increasing reflectivity of the OC. This wavelength red-shift is caused by the enhanced reabsorption effect with low OC transmission, where a lower population inversion is needed, resulting in a drift of gain spectrum. Note that the two emission peaks for $T_{\mathrm{oc}}=72 \%$ could be attributed to the same gain crosssections in this case.

To evaluate the propagation loss of the SCF, Caird analysis that was modified for the case of high $T_{\mathrm{oc}}$ was performed ${ }^{[18]}$. The dependence of the slope efficiency $\eta$ on $T_{\mathrm{oc}}$ and the internal loss $L$ per pass could be expressed as ${ }^{[19]}$

$$
\frac{1}{\eta}=\frac{1}{\eta_{0}\left(1+\frac{2 \gamma}{\gamma_{\mathrm{oc}}}\right)},
$$

where $\eta_{0}$ is an intrinsic slope efficiency, $\gamma=-\ln (1-L)$ and $\gamma_{\mathrm{oc}}=-\ln \left(1-T_{\mathrm{oc}}\right)$. Note that the emission wavelength and the corresponding stimulated emission crosssection were regarded as constant since the laser wavelength depended only weakly on $T_{\text {oc }}$ (only $5 \mathrm{~nm}$ drift as shown in Figure 2(b)). Figure 3 shows the inverse slope efficiency versus the inverse output-coupling loss. Best fit of the experimental data yielded $\eta_{0} \approx 60 \%$ and a propagation loss

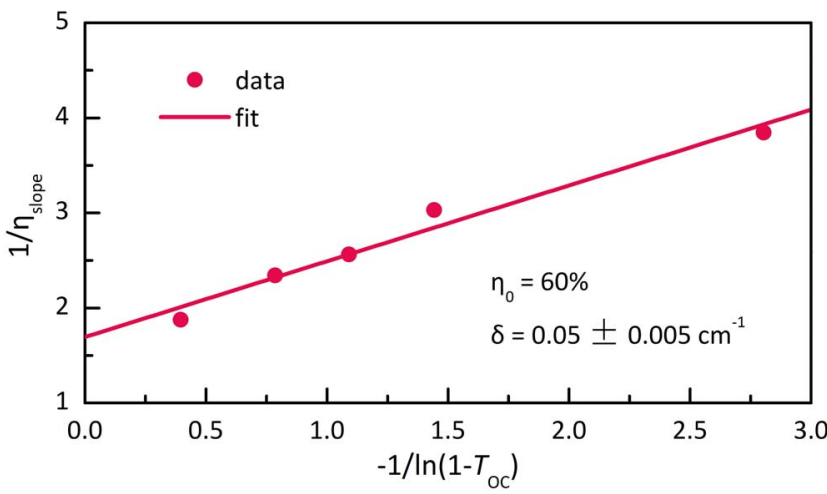

Figure 3. Caird plot for the high-power Ho:YAG SCF laser: inverse slope efficiency with respect to the inverse output-coupling loss, i.e., $-\ln \left(1-T_{\mathrm{oc}}\right)$.
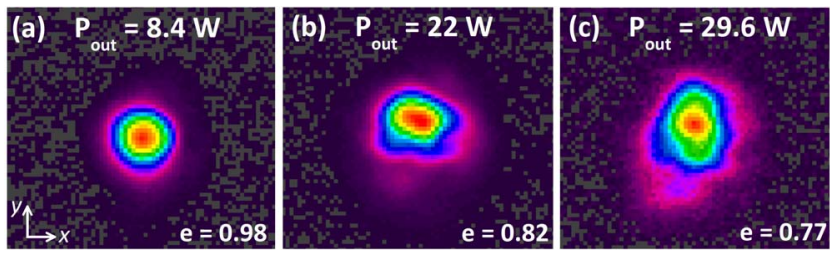

Figure 4. Beam intensity profiles recorded at different output powers with $T_{\mathrm{oc}}=72 \% ; e$ is the calculated beam ellipticity from each image.

at the laser wavelength of $\delta=L / l=0.05 \pm 0.005 \mathrm{~cm}^{-1}$. The calculated limiting slope efficiency is considerably less than the quantum efficiency of $\sim 91 \%$, which is caused by the following two reasons: first, the noncollinear pumping configuration, i.e., about $2^{\circ}$ between the pump and laser beams at the front facet of the SCF used to avoid the unwanted feedback to the Tm-fiber pump laser, and second, the energy-transfer upconversion of $\mathrm{Ho}^{3+}$ ions in the ${ }^{5} \mathrm{I}_{7}$ manifold under pump bleaching ${ }^{[20]}$. The propagation loss is larger than the previously reported value of $0.02 \mathrm{~cm}^{-1}$, 'directly measured' in a $400 \mu$ m-diameter Ho:YAG SCF fabricated by LHPG method ${ }^{[21]}$. Anti-reflection coating on the SCF and optimizing the crystal quality would decrease the overall losses, thus contributing to further scaling of the laser power.

Far-field beam intensity profiles of the Ho:YAG SCF laser with $T_{\mathrm{oc}}=72 \%$ were recorded at different output powers by using a CCD camera (PY-III-HR-C-A, Ophir). As shown in Figure 4(a), the beam profile at $8.4 \mathrm{~W}$ output power was a perfect circle with a beam ellipticity (defined as a ratio of the beam diameters in the $x$ - and $y$-directions) of $e=0.98$. This implies uniform thermal distribution over the crosssection of the SCF owing to the large surface area-to-volume ratio. The corresponding near-field beam profile presented a similar intensity distribution which was obviously not affected by the noncircular shape of the SCF, indicating a free-space propagation of the laser beam in the SCF. This could be further confirmed by calculating the beam size on 
the end facet of the SCF with $T_{\mathrm{oc}}=92 \%$ for the case when the end facet itself was employed as the output coupler. Using a $2 f$ imaging system and knife-edge technique, the beam diameter was roughly estimated to be $300 \mu \mathrm{m}$ on the SCF end facet, which equals the simulated value using the ABCD-matrix analysis. When the output power reached $22 \mathrm{~W}$, as shown in Figure 4(b), the beam shape was no more circular because of the generation of high-order modes, giving an $e$ value of 0.82 . The excitation of high-order modes is easily understood since the noncollinear pumping configuration and no additional transverse-mode-limiting optical elements were applied. Further power scaling to nearly $30 \mathrm{~W}$ produced a deteriorated beam profile (see Figure 4(c)) with an elliptic shape $(e=0.77)$. The smaller central intensity distribution (the area of the red part) is an indication of thermal lens effect and the asymmetric thermal distribution over the transverse cross-section of the SCF. To mitigate the thermal issues, longer SCF with lower active ion doping level will be preferable for further improving the laser performance. Moreover, direct water cooling around the SCF will mitigate thermal effect and favor uniform thermal diffusion, since the nonoptimized thermal contact is responsible for the main thermal difference between the mount and SCF center ${ }^{[22]}$. Nevertheless, the thermal issue in present work is more mitigated than in the previous report in which the $\mathrm{CW}$ laser power began to roll off at $10 \mathrm{~W}$ output $^{[15]}$

The beam propagation factors $\left(M^{2}\right)$ at different absorbed pump powers were measured using the same CCD camera. As depicted in Figure 5(a), the measured $M^{2}$-factors in both $x$ - and $y$-directions were around 1.1 at low pump levels, i.e., $<20 \mathrm{~W}$ absorbed pump power. However, excitation of high-order transverse modes when further increasing the pump power would gradually degrade the beam quality. On the other side, beam quality along the $x$-axis exhibits a stronger deterioration than that along the $y$-axis, which is attributed to the unidirectional heat dissipation in the SCF at high power levels. At $25 \mathrm{~W}$ of absorbed pump power, the $M^{2}$-factors along the $x$ - and $y$-directions were measured to be 1.32 and 1.24 , respectively. The $M^{2}$-factors were not measured at higher output powers because of the obvious multi-mode emission. Figure 5(b) shows a typical $M^{2}$-factor measurement at an output power of $7 \mathrm{~W}$ corresponding to an absorbed power of $15 \mathrm{~W}$. In this case, the measured $M^{2}$ factor is comparable with the value of 1.1 that was obtained by employing a Ho:YAG thick rod ${ }^{[23]}$.

\section{Conclusion}

In conclusion, we have experimentally demonstrated a highpower Ho:YAG SCF laser inband pumped by a Tm-fiber laser at $1908 \mathrm{~nm}$. With a modified Caird analysis, the propagation loss coefficient of this Ho:YAG SCF was estimated to
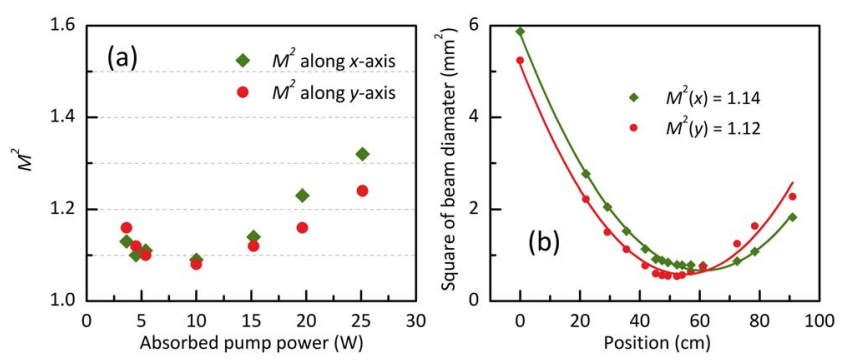

Figure 5. (a) The measured $M^{2}$-factor of the Ho:YAG SCF laser $\left(T_{\mathrm{oc}}=\right.$ $92 \%$ ) at different absorbed pump powers and (b) a typical $M^{2}$ measurement at $7 \mathrm{~W}$ output laser power.

be $0.05 \pm 0.005 \mathrm{~cm}^{-1}$. A maximum output power of $35.2 \mathrm{~W}$ was achieved with $T_{\mathrm{oc}}=72 \%$, corresponding to a slope efficiency of $42.7 \%$. Without the traditional spatial mode matching, excitation of high-order transverse modes seems unavoidable under high powers, which will result in a serious deterioration of the beam quality. Nevertheless, the measured $M^{2}$-factors are below 1.2 for output powers up to $10 \mathrm{~W}$. Thermal lens effects were obviously enhanced at a power level approaching $\sim 30 \mathrm{~W}$. Therefore, a longer SCF with a lower doping level and direct water cooling will be essential for further power scaling and improving the beam quality, whereas anti-reflection coating and limitation of the highorder transverse modes are expected to improve the overall laser performance.

The present work is a demonstration of the high quality of the fabricated Ho:YAG SCF. Amplification of ultrashort pulses (including picosecond and femtosecond) near $2 \mu \mathrm{m}$ to high average/peak power will be the next step, relying on advantageous features such as the high laser damage threshold, straightforward thermal management (large surface-tovolume ratio), excellent thermo-mechanical properties and weak nonlinear effects. Moreover, the encountered problems such as the excitation of high-order transverse modes and thermal lens effect at high power levels would be absent or effectively compensated by external lens when the SCF is employed as an amplifier.

\section{Funding}

Foundation of Deutscher Akademischer Austauschdienst (DAAD) - Research Stays for University Academics and Scientists (57381327); Natural Science Foundation of Jiangsu Province, China (SBK2019030177); China Scholarship Council (201704910363); Foundation of the Government of the Russian Federation (074-U01).

\section{Acknowledgement}

Y. Zhao acknowledges financial support from the Alexander von Humboldt Foundation through a Humboldt fellowship. 


\section{References}

1. S. W. Henderson, C. P. Hale, J. R. Magee, M. J. Kavaya, and A. V. Huffaker, Opt. Lett. 16, 773 (1991).

2. C. Gottlob, G. E. Kopchok, S.-K. Peng, M. Tabbara, D. Cavaye, and R. A. White, Lasers Surg. Med. 12, 86 (1992).

3. T. Sumiyoshi, H. Sekita, T. Arai, S. Sato, M. Ishihara, and M. Kikuchi, IEEE J. Sel. Top. Quantum Electron. 5, 936 (1999).

4. U. N. Singh, B. M. Walsh, J. Yu, M. Petros, M. J. Kavaya, T. F. Refaat, and N. P. Barnes, Opt. Mater. Express 5, 827 (2015).

5. A. Dergachev, D. Armstrong, A. Smith, T. Drake, and M. Dubois, Opt. Express 15, 14404 (2007).

6. T. Walbaum, M. Heinzig, T. Schreiber, R. Eberhardt, and A. Tünnermann, Opt. Lett. 41, 2632 (2016).

7. S. D. Jackson, A. Sabella, A. Hemming, S. Bennetts, and D. Lancaster, Opt. Lett. 32, 241 (2007).

8. M. N. Zervas and C. A. Codemard, IEEE J. Sel. Top. Quantum Electron. 20, 0904123 (1999).

9. X. Délen, A. Aubourg, L. Deyra, F. Lesparre, I. Martial, J. Didierjean, F. Balembois, and P. Georges, Proc. SPIE 9342, 934202 (2015).

10. V. Markovic, A. Rohrbacher, P. Hofmann, W. Pallmann, S. Pierrot, and B. Resan, Opt. Express 23, 25883 (2015).

11. X. Délen, Y. Zaouter, I. Martial, N. Aubry, J. Didierjean, C. Hönninger, E. Mottay, F. Balembois, and P. Georges, Opt. Lett. 38, 109 (2013).

12. X. Délen, I. Martial, J. Didierjean, N. Aubry, D. Sangla, F. Balembois, and P. Georges, Appl. Phys. B 104, 1 (2011).
13. X. Délen, S. Piehler, J. Didierjean, N. Aubry, A. Voss, M. A. Ahmed, T. Graf, F. Balembois, and P. Georges, Opt. Lett. 37, 2898 (2012).

14. A. M. Rodin and M. Zopelis, in Conference on Lasers and Electro-Optics Pacific Rim (CLEO-PR) (2017), p. 1.

15. Y. Li, K. Miller, E. G. Johnson, C. D. Nie, S. Bera, J. A. Harrington, and R. Shori, Opt. Express 24, 9751 (2016).

16. J. Wang, Q. Song, Y. Sun, Y. Zhao, W. Zhou, D. Li, X. Xu, C. Shen, W. Yao, L. Wang, J. Xu, and D. Shen, Opt. Lett. 44, 455 (2019).

17. Y. Wang, R. Lan, X. Mateos, J. Li, C. Hu, C. Li, S. Suomalainen, A. Härkönen, M. Guina, V. Petrov, and U. Griebner, Opt. Express 24, 18003 (2016).

18. J. Morris, N. K. Stevenson, H. T. Bookey, A. K. Kar, C. T. A. Brown, J.-M. Hopkins, M. D. Dawson, and A. A. Lagatsky, Opt. Express 25, 14910 (2017).

19. P. Loiko, R. Soulard, G. Brasse, J. Doualan, B. Guichardaz, A. Braud, A. Tyazhev, A. Hideur, and P. Camy, Opt. Express 26, 24653 (2018).

20. N. P. Barnes, B. M. Walsh, and E. D. Filer, J. Opt. Soc. Am. B 20, 1212 (2003).

21. Y. Li, E. G. Johnson, C. D. Nie, J. A. Harrington, and R. Shori, Opt. Express 22, 14896 (2014).

22. D. Sangla, I. Martial, N. Aubry, J. Didierjean, D. Perrodin, F. Balembois, K. Lebbou, A. Brenier, P. Georges, O. Tillement, and J. M. Fourmigue, Appl. Phys. B 97, 263 (2009).

23. D. Y. Shen, A. Abdolvand, L. J. Cooper, and W. A. Clarkson, Appl. Phys. B 79, 559 (2004). 